\title{
Plant viruses of the Amalgaviridae family evolved via recombination between viruses with double-stranded and negative-strand RNA genomes
}

Mart Krupovic $^{1^{*}}$, Valerian V Dolja ${ }^{2}$ and Eugene V Koonin ${ }^{3^{*}}$

\begin{abstract}
Plant viruses of the recently recognized family Amalgaviridae have monopartite double-stranded (ds) RNA genomes and encode two proteins: an RNA-dependent RNA polymerase (RdRp) and a putative capsid protein (CP). Whereas the RdRp of amalgaviruses has been found to be most closely related to the RdRps of dsRNA viruses of the family Partitiviridae, the provenance of their $\mathrm{CP}$ remained obscure. Here we show that the $\mathrm{CP}$ of amalgaviruses is homologous to the nucleocapsid proteins of negative-strand RNA viruses of the genera Phlebovirus (Bunyaviridae) and Tenuivirus. The chimeric genomes of amalgaviruses are a testament to the effectively limitless gene exchange between viruses that shaped the evolution of the virosphere.
\end{abstract}

Reviewers: This article was reviewed by Lakshminarayan M. lyer and Nick V. Grishin. For complete reviews, see the Reviewers' Reports section.

Keywords: Amalgaviridae, Virus origin, dsRNA viruses, Negative-sense RNA viruses, Capsid proteins

\section{Findings}

Eukaryotes host a variety of RNA viruses which have positive-strand genomes (virions contain RNA of the same polarity as mRNA), negative-strand genomes (virions contain RNA complementary to the mRNA) or dsRNA genomes [1,2]. These viruses prey on hosts from all major eukaryotic supergroups and are extremely diverse both genetically and structurally, making it difficult to trace their origins. Genetic recombination, which often leads to the emergence of new chimeric entities, dramatically complicates the reconstruction of the evolutionary history of RNA viruses [3,4]. One approach to delineate the underlying evolutionary relationships is based on phylogenetic analysis of viral RNA-dependent RNA polymerases $(\mathrm{RdRp})$, the only protein universally present in all non-defective RNA viruses [2,5]. A complementary

\footnotetext{
* Correspondence: krupovic@pasteur.fr; koonin@ncbi.nlm.nih.gov 'Department of Microbiology, Unité Biologie Moléculaire du Gène chez les Extrêmophiles, Institut Pasteur, Paris 75015, France

${ }^{3}$ National Center for Biotechnology Information, National Library of Medicine, National Institutes of Health, Bethesda, MD 20894, USA

Full list of author information is available at the end of the article
}

strategy focuses on comparison of viral proteins responsible for virion formation, the hallmark of viruses [6-11]. It is becoming increasingly clear that only a combination of the two approaches can capture the full extent of evolutionary connections among different groups of viruses and other types of selfish genetic elements [12,13].

The evolution of viruses with dsRNA genomes appears to be particularly convoluted. In all likelihood, this class of viruses is polyphyletic: some groups appear to have evolved from different lineages of eukaryotic positivestrand RNA viruses, whereas others have apparently emerged from dsRNA bacteriophages [5,14-18]. Recently, a new group of dsRNA viruses has been described and classified into the family Amalgaviridae. These viruses have been isolated from various plants in the form of dsRNA molecules of $3.5 \mathrm{~kb}$ [19-22]. The genomes uniformly encode two predicted proteins, the $\mathrm{RdRp}$ and a putative capsid protein (CP). Such bicistronic genome organization is characteristic of dsRNA viruses of the family Totiviridae which infect fungi and protists [23]. However, phylogenetic analyses have shown that the amalgavirus RdRps form a sister clade to the corresponding 
proteins of partitiviruses (Partitiviridae) which have segmented (bipartite) dsRNA genomes and infect plants, fungi and protists (Additional file 1: Figure S1) [20-22]. Consequently, it has been suggested that amalgaviruses "amalgamate" features of totiviruses and partitiviruses (hence the family name) and represent an intermediate between the two viral groups [20]. However, both totiviruses and partitiviruses form icosahedral virions [23,24], whereas in the case of amalgaviruses, all attempts to visualize virus particles have so far failed [20-22]. This result was suggested to signify either low titer of amalgaviral particles or lack of bona fide viral particles altogether, as is the case for hypoviruses and endornaviruses [22]. Indeed, the putative $\mathrm{CP}$ of amalgaviruses shows no significant sequence similarity to the CPs of totiviruses or partitiviruses, and sequence analyses of this protein previously offered no clues as to its provenance. Immuno-gold labeling has shown that the putative $\mathrm{CP}$ is expressed during viral infection and is found within amorphous bodies in the cytoplasm [25]. Here we trace the source of the putative amalgaviral $\mathrm{CP}$ to negative-strand RNA viruses and propose an evolutionary scenario for the origin of these unusual dsRNA viruses.

BLASTp searches seeded with sequences of CPs from the four currently known amalgaviruses, Southern tomato virus (STV [22]), Vicia cryptic virus M (VCV-M [19]), Blueberry latent virus (BBLV [20]), and Rhododendron virus A (RdVA [21]), did not reveal any relationship to proteins of other viruses, consistent with previous attempts. However, several CP homologs were detected in the genomes of various plants, including Populus trichocarpa, Medicago truncatula and Theobroma cacao. In P. trichocarpa, the CP homolog was found next to a truncated BBLV-like RdRp gene (XP_002310263 matches both $\mathrm{CP}[\mathrm{E}=6 \mathrm{e}-09]$ and $\mathrm{RdRp}[\mathrm{E}=7 \mathrm{e}-25]$ of $\mathrm{BBLV})$, pointing towards occasional, probably spurious integration of amalgavirus sequences into the host DNA and suggesting that the host range of amalgaviruses might be considerably broader than currently known.

Amalgaviral CPs are highly divergent and share only 19-25\% sequence identity (with the closest pair being RhVA-STV; Figure 1A). The common feature of these proteins is high content of predicted $\alpha$-helices and near complete absence of $\beta$-strands [20-22]. To search for remote homologs of amalgaviral CPs, we employed HHpred [26] which performs pairwise comparison of hidden Markov model profiles and takes into account the experimentally determined or predicted secondary structures of the compared proteins. Unexpectedly, searches seeded with the putative CP of STV returned hits to the nucleocapsid (NC) proteins of animal-infecting phleboviruses (genus Phlebovirus, family Bunyaviridae) and plant tenuiviruses (unassigned genus Tenuivirus). Both phleboviruses and tenuiviruses have segmented negative-sense RNA genomes that are complexed with the NC proteins to form ribonucleoprotein (RNP) filaments [27,28]. Whereas phleboviral RNPs are packed into spherical membrane-bound virions, the RNPs of tenuiviruses are not further encapsidated and persist as non-enveloped filamentous structures that can adopt circular or branched configurations [28]. Structural analyses of NC proteins from several phleboviruses have revealed a unique $\alpha$-helical fold consisting of two domains: the core domain involved in sequence unspecific RNA binding and the flexible helical arm domain responsible for protein oligomerization [29-31]. The NC is unique to viruses from these two genera and thus far has not been reported for any other virus group. Besides the $\mathrm{NC}$, phleboviruses and tenuiviruses share several other proteins, and in phylogenetic analyses of the RdRps, tenuiviruses cluster with phleboviruses, deeply within the family Bunyaviridae, suggesting that tenuiviruses have evolved from phleboviruses [28].

The region matched by HHpred between STV CP and phleboviral/tenuiviral NCs, extended over 216 residues (from 52 to 266) of the STV protein (Figure 1B) and encompassed most of the phleboviral NC protein sequence (197 of 248 residues). Although statistical significance of the obtained hits was not particularly high ( $\mathrm{P}=71.8 \%$; Additional file 2: Figure $\mathrm{S} 2$ ), we pursued this lead and investigated the relationship in more detail. To validate the potential homology of amalgaviral CPs and phleboviral/tenuiviral NCs, a representative set of phleboviral/tenuiviral NC sequences was downloaded from the PFAM database (Family: Tenui_N; PF05733) and aligned with the proteins of the four amalgaviruses. Notably, tenuiviral NC sequences were as similar to the phleboviral homologs as they were to amalgaviral CPs (Figure 1A). Due to the high divergence of amalgaviral CPs, multiple sequence alignments were constructed between phleboviral/tenuiviral NCs and individual CPs of amalgaviruses. As can be judged from the sequence alignment shown in Figure 1D, amino acid positions conserved between tenuiviruses and phleboviruses are also conserved in the CP of amalgavirus RhVA. Furthermore, the predicted secondary structure profile of the RhVA CP is strongly similar to the experimentally determined structure of the NC of Severe fever with thrombocytopenia syndrome virus (Phlebovirus), despite several insertions in the former protein (Figure 1D). It should be noted that, the high sequence divergence notwithstanding, the secondary structure profiles are closely similar for all four amalgaviral CPs (Additional file 3: Figure S3). Analysis of the sequence conservation distribution in the context of the tertiary structure of the phleboviral NC shows that the conserved residues are evenly distributed within the 'core' domain of the protein (Figure 1C). Notably, the three positively charged residues, which have 


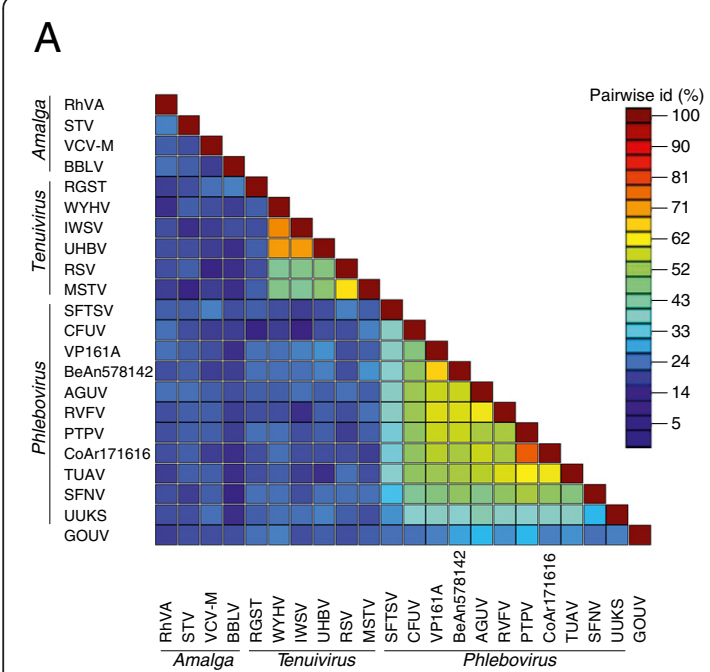

B
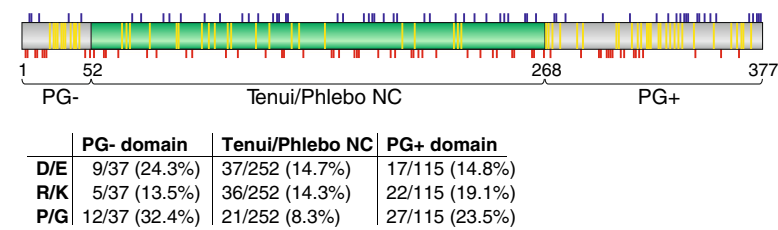

C

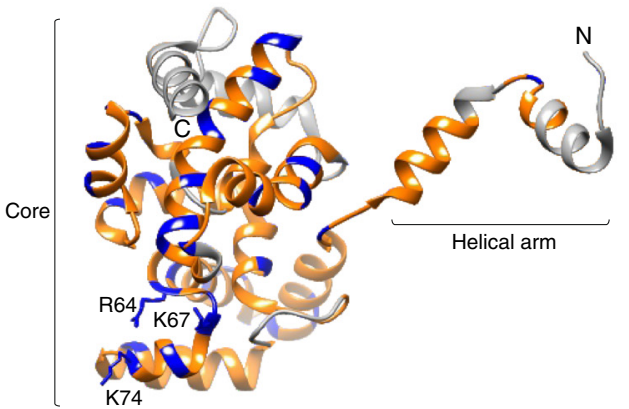

D

ss_RhVA (Jpred)

Ss_SFTSV (PDB:4J4R)

E1B261IRhVA

4J4RISFTS

3ov9IRVFV

F4ZCK3IAGUV

A7KCNGIPTPV

A7KCN8ICo Ar 171616 9 QFASES---IDEQAVAGWVTD -

F2W3T5ITUAV 17161699 HFASES--- IDESTISAWVSD -

10 ELAGHE---VDTATISAWVQA-

A7KCJGIBe An 57814210 EFSGEA--- INLAE IQGWVTD--

$\begin{array}{lll}\text { A7KCK4IVP-161A } & 10 & \text { AFAGEP--- INNAEVMGWVNE } \\ \text { A7KCM8ISFNV } & 12 & \text { AFAEES---ADVSTIESWVND } \\ \text { AKCCKBCFV } & & \end{array}$

A7KCK8ICFUV 10 EFGEQA--- IDETVVQEWLQN --

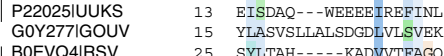

P27207IMSTV

25
25
SYLTAH-----KADVVTFAGQ

P84254IWYHV

20 KYISEN-----KDSLVVFVGQ -

O11440IUHB

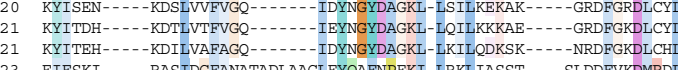

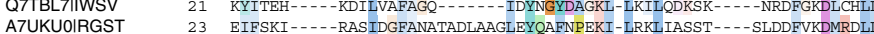

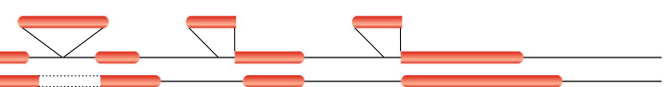

Ss_RhVA (Jpred)

ss_SFTSV (PDB:4J4R)

E1B261IRhVA

4J4RISFTSV
3ov9IRVFV

F4ZCK3IAGUV

A7KCN6IPTPV

VYEAGMPAGRFTVQRVHEI

F- (34) -VIRW

RWARSN - (10) -KVKKVED-KRA- (16) -QQGIMAEEVKEARASTQ- - - - - 179 - LAYEGLDPALI I IKKLKETG- - - - - GDDWVKDTKFI I - - - - -VEALTR GN- - - - -KIVKASG-KMS - - - - NSG- -SKRLMALQEKYGLVERAE- -T 101

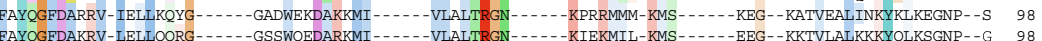

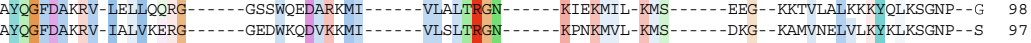
AYQGFDAKRV- IAL IKERG - - - - - GENWKEDVKKMI - - - --VLSLTRGN - - - --KPAKMIL-KMS - - - --DKG- -KKEVNDLITRYKLKSGNP- - S 97 FAYQGFDARRV - IELIKLRG - - - - GDNWMEDAKQMI - - - - - ILCLTRGN- - - - KPTKMMA-KMS - - - - EKG - KKIVQGLIKKYNLKEGNP - -S 98 FAYQGFDARRI-VELVQQKG- - - - - GAGWKDDVKMMI - - - - - VLCLTRGN- - - - KPTKKMVE-KMS- - - - - PEG- -KVKVNRLISTYGLKSGNP- - G 98 FAYEGFNAQRI - IQLVQEKG- - - - PQTWQTDVKMMI - - - - - VLALTRRGN- - - - KPSKMIE-KMS - - - - AEG- -KKKASRLITIYGLKSGNP- - G 98

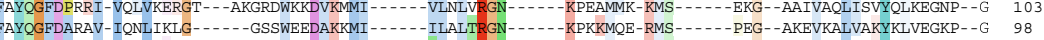
QYQGFDAAVV-LSRIFELAKKADLSRDQMLRDIRALI - - - - - TLLHLTRGN - - - - - -KLSSIEK-RLS - - - - - EEG- - KKEFALIKARYQLVDKAK- -E 107 LEYQGFDPYAF-LSYLFAVAKKAGIGEAEHKKNLQTLA- - - - - VLGTMRGG- - - - KAKKIAE-KST- - - - - PET- - KRWLESMIQKYSITSGRPT-G 113 - IEYAGYDAATL-IGILKDKG - - - - - GDTLARDMTMCI - - - - - TMRYVRGT- - - - - GFVRDVTKKVK- - (1) -AAG- -STEASTLVSRYGIVSSVGT -N 114

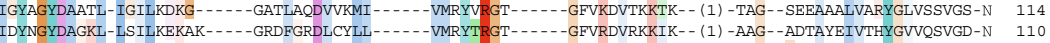
TGYDAGKL - LKILODKSK - - - NRDFGIDLCHLL $\begin{array}{lll} & \text { VMRYTRGT - - - - GFVRDVRKKIK- - (1) - AAG- - GDTAHEIVTHYGVVHSVGD -N } & 111 \\ \end{array}$ - VMRYTRGT-----GFVRDVRKKIK-- (1) -AAG- -GETSHEIVTHYGIVQSVGD-N 111

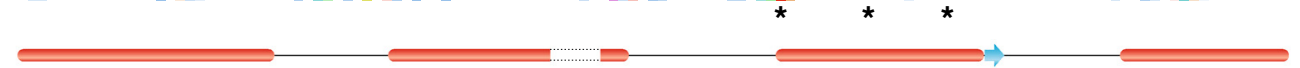

Figure 1 (See legend on next page.) 
(See figure on previous page.)

Figure 1 Relationship between the putative capsid proteins of amalgaviruses and nucleocapsid proteins of tenuiviruses and phleboviruses. A. Pairwise identity plot of capsid and nucleocapsid proteins of amalgaviruses, tenuiviruses and phleboviruses. Protein accession numbers are provided in panel D, whereas the full virus names are provided in the Abbreviations section. B. Schematic representation of the putative capsid protein of rhododendron virus A (RhVA). The proline/glycine-rich N- and C-terminal domains (PG-and PG+, respectively) are shown in grey, whereas the central domain showing similarity to the nucleocapsid proteins of phleboviruses and tenuiviruses (Tenui/Phlebo NC) is in green. Blue and red ticks above and below the scheme indicate the positions of positively (Arg, Lys) and negatively (Asp, Glu) charged amino acid residues, respectively, whereas yellow streaks correspond to Pro and Gly residues. The table includes the counts and percentages of Asp/Glu (D/E), Arg/Lys $(\mathrm{R} / \mathrm{K})$ and Pro/Gly $(\mathrm{P} / \mathrm{G})$ residues in the respective domains. C. X-ray structure of the nucleocapsid protein of Severe fever with thrombocytopenia syndrome virus (SFTSV). Amino acid residues shared with RhVA CP are shown in blue, regions that have counterparts in RhVA but do not show close sequence similarity are shown in orange, and regions that are absent in the RhVA protein are shown in grey. The residues important for RNA-binding are shown with ball-and-stick representation. The 'Core' and 'Helical arm' domains are indicated. D. Multiple sequence alignment of RhVA CP with tenuiviral and phleboviral NC homologs. Sequences are identified with UniProt or PDB accession numbers followed by abbreviated virus names. Above the alignment are the predicted (for RhVA) and experimentally determined (for SFTSV) secondary structure elements; a-helices, red ellipses; $\beta$-strands, blue arrows. The alignment is colored according to sequence conservation using the standard Clustal color scheme.

been found to play an essential role in RNA binding by the Rift Valley fever virus NC [29], are conserved in the RhVA protein. However, certain degree of variation is observed in this region in CPs of other amalgaviruses (Additional file 3: Figure S3), as is the case for tenuiviral NCs (Figure 1D).

The major differences between the amalgaviral CPs both within the family (Additional file 3: Figure S3) as well as when compared to the phleboviral/tenuiviral NCs are localized to the $\mathrm{N}$ and $\mathrm{C}$ termini. In particular, RhVA CP, which is the largest among amalgaviral CPs (404 aa), lacks the regions corresponding to the first of the two $\alpha$-helices within the N-terminal helical arm domain and the two C-terminal $\alpha$-helices (Figure 1C). The three $\alpha$-helices ( $\alpha 1, \alpha 12$ and $\alpha 13$ in Rift Valley fever virus NC) are located at the periphery of the hexameric NC ring, and residues projecting from these helices have been proposed to form a large part of the RdRp-binding surface [29]. Given that the RdRps of amalgaviruses and phleboviruses are highly dissimilar, it seems likely that the changes within the $\mathrm{N}$ - and C-terminal regions of the amalgaviral CPs were driven by the necessity to adapt to the partitivirus-like RdRp (Additional file 1: Figure S1). Both the $\mathrm{N}$-terminal and the $\mathrm{C}$-terminal regions in the RhVA CP are rich in proline and glycine residues. Furthermore, when compared to the central domain, which bears similarity to the phleboviral/tenuiviral NCs, the N-terminal region is enriched in negatively charged amino acids, whereas the $\mathrm{C}$-terminal region is more positively charged (Figure 1B). Notably, the C-terminal region of the tenuiviral $\mathrm{NC}$ is also enriched in positively charged residues and has been shown to bind nucleic acids [32]. Consequently, although $\mathrm{N}$ - and $\mathrm{C}$-terminal regions display considerable variation among the amalgaviral CPs (Additional file 3: Figure S3), they might play important roles in mediating various protein-protein and protein-RNA interactions.

\section{Conclusions}

It has been proposed that amalgaviruses share features with partitiviruses and totiviruses and thus might represent an evolutionary link between the two virus groups [22]. Our results indicate that the $\mathrm{CP}$ of amalgaviruses evolved from the $\mathrm{NC}$ of phleboviruses or tenuiviruses. Considering that tenuiviruses replicate in plants, they appear as a more likely source of the $\mathrm{NC}$ gene of amalgaviruses. We propose that the ancestor of amalgaviruses has emerged by recombination between a partitivirus, which contributed the $\mathrm{RdRp}$ gene, and a tenuivirus which donated the $\mathrm{NC}$ gene. In partitiviruses, the RdRp is the sole gene encoded in one of the two genomic segments [24]. Thus, incorporation of the NC gene into such genomic segment would conceivably lead to size increase of the resultant chimeric genome, precluding its incorporation into the original partitivirussized capsids. An outstanding question is the exact role of the CP in amalgaviruses. Phleboviral/tenuiviral NCs are known to bind ssRNA [31], whereas the genomes of amalgaviruses are thought to consist of dsRNA. On the one hand, it cannot be ruled out that following the acquisition from tenuiviruses, the CPs have evolved the ability to bind dsRNA instead of ssRNA. Indeed, phleboviral NCs have an inherent ability to form diverse oligomers and bind both ssRNA and ssDNA [29,31], whereas $\mathrm{NC}$ of tenuiviruses can also bind dsDNA [32]. On the other hand, amalgaviral CPs might interact with the single-stranded form of the genome. Given that dsRNA is the necessary intermediate that is always produced upon replication of both ssRNA and dsRNA viruses, the dsRNA genomes of amalgaviruses isolated directly from the plant tissues might represent a replicative intermediate rather than the "encapsidated" form of the genome. Another possibility is that the $\mathrm{CP}$ of amalgaviruses was recruited for a function distinct from the original role of the tenuivirus NC. For example, the NC of plantinfecting rhabdoviruses, besides encapsidating the viral genomic RNA, is a component of the viroplasms $[28,33]$. Amorphous bodies immuno-labeled with antibodies against BBLV CP in the cytoplasm of BBLV-infected cells could actually represent such viroplasm-like structures. 
Efficient vertical transmission of the amalgaviruses through the seed, together with their inability to mechanically transmit from plant-to-plant [20-22], are compatible with the apparent lack of the bona fide extracellular virions that are normally required for the latter transmission route. Regardless of the precise roles of the NC-derived protein in virus reproduction, amalgaviruses represent a remarkable case of transfer of viral hallmark genes between widely different RNA viruses and together with similarly striking examples of gene exchange between viruses with RNA and DNA genomes [34-36], emphasize the ultimate modularity of the virosphere.

\section{Methods}

Sequences of the putative capsid proteins of amalgaviruses were obtained from GenBank and their homologs in viral and cellular genomes were searched for using BLASTp [37]. Distant homology detection was performed using HHpred [26]. Sequences of phleboviral and tenuiviral nucleocapsid proteins (family: Tenui_ $N$ ) were downloaded from the PFAM database (PF05733) and clustered to $80 \%$ sequence identity using BLASTclust at http://toolkit.tuebingen.mpg.de/blastclust. Protein sequences were aligned using Promals3D [38] and visualized using Jalview [39]. Secondary structure of the rhododendron virus A capsid protein was predicted using Jpred [40]. All-against-all pairwise identities were calculated using Sequence Demarcation Tool [41]. X-ray structure of the Severe fever with thrombocytopenia syndrome virus was downloaded from the Protein Data Bank (4J4R) [30] and rendered using UCSF Chimera [42]. Maximum likelihood phylogenetic analysis of RdRps was carried out by using PhyML 3.1 [43], with the JTT model of amino acid substitution, including a gamma law with 4 substitution rate categories, and an estimated proportion of invariable sites.

\section{Reviewers' reports}

Reviewer 1: Lakshminarayan M. Iyer, National Center for Biotechnology Information, NIH, Bethesda

This is an interesting twist on the origins of the Amalgaviridae and proposes their derivation from a recombination between a partitivirus and a tenuivirus. The study hinges on the unification of the Amalgaviral Capsid proteins with the Nucleocapsid proteins of negative strand RNA viruses such as Tenuviruses and Pheloboviruses using Profile-profile searches. I can reproduce their search results, although searches only retrieves the NC proteins from some starting points and alignment queries do not retrieve NC proteins in profile-profile searches. The statistics is also very weak. I would have been very hesitant if I were to make this conclusion. This is primarily because in my experience profile-profile searches might often retrieve unrelated alpha-helical proteins at low confidence values when alpha-helical proteins are used as query and one could be biased by the names of the retrieved proteins. However, to credit the authors, they have compared conservation of sequence and structure and have considered the structure of the core to reach their conclusions with very convincing arguments. The paper is well written otherwise and can be published without any modifications.

Authors' response: We appreciate this expert assessment.

\section{Reviewer 2: Nick V. Grishin, University of Texas Southwestern Medical Center, Dallas}

In this interesting, thoroughly-executed and well-written study, the authors successfully trace evolutionary origins of one of the two protein encoded by Amalgaviridae. Using best available sequence-based methods augmented with expert analysis of alignments, they find homologs of this protein in better-studied viruses and thus predict its 3D structure. This prediction looks very reasonable and has several interesting biological ramifications. First, since the homologs of this protein are capsid-forming (or nucleic-acid-packaging) proteins, this homology provides additional evidence that Amalgaviridae possess a capsid protein. Second, this homology suggests the origin of these viruses by recombination of genomes of two different groups of viruses. Third, it implies that unusual alpha-helical capsids may be more widespread than previously thought. More generally, finding homologs for viral proteins is very difficult due to high numbers of fixed amino acid substitutions leading to large sequence divergence between homologs. This is a well-known problem, and it is addressed very nicely in this paper. I. e., a careful study of weekly similar sequence or threading hits could be very productive.

Authors' response: We appreciate the expert appraisal of the article.

Minor technical suggestions:

"recently established family" is not very clear, it may be understood as a family that originated recently in evolution. Maybe something like "recently described" or "recently erected" or smth similar.

Authors' response: good point, 'established' changed to 'recognized'.

Due to its more speculative nature, should this section be called "Discussion" instead?

Authors' response: We appreciate the suggestion but the Discovery Notes format mandates Conclusions following the Findings section. Hopefully, this is interpreted as intended, i.e. as "Discussion and Conclusions".

\section{Additional files}

Additional file 1: Figure S1. Maximum likelihood tree of the RdRp proteins from diverse RNA viruses. The tree was rooted on the branch of positive-strand RNA viruses (Picornaviridae and Caliciviridae). Numbers at 
the branch points represent SH-like local support values. Branches with support values below $50 \%$ were collapsed. The scale bar represents the number of substitutions per site.

Additional file 2: Figure S2. Results of the HHpred search seeded with the putative capsid protein of Southern tomato virus (YP_002321510). $H(h)$, a-helix; C(c), coil.

Additional file 3: Figure S3. Multiple sequence alignment of amalgaviral capsid proteins. All sequences are indicated with their GenBank identifiers followed by abbreviated virus names. Positively charged amino acid residues predicted to be involved in RNA binding are shown in bold and underlined. The last two lines in each block show consensus amino acid sequence (Consensus_aa) and consensus predicted secondary structures (Consensus_ss). The protein sequences are colored according to predicted secondary structures (red: alpha-helix, blue: beta-strand). Consensus predicted secondary structure symbols: alpha-helix: h; beta-strand: e. Consensus amino acid symbols are: conserved amino acids are in uppercase letters; aliphatic (I, V, L): I; aromatic (Y, H, W, F): @; hydrophobic (W, F, Y, M, L, I, V, A, C, T, H): h; alcohol $(S, T)$ : o; polar residues $(D, E, H, K, N, Q, R, S, T)$ : p; tiny $(A, G, C, S)$ : t; small ( $A, G, C, S, V, N, D, T, P): s$; bulky residues $(E, F, I, K, L, M, Q, R, W, Y)$ : b; positively charged $(K, R, H)$ : +; negatively charged $(D, E)$ : -; charged $(D, E, K$ R, H): c. The alignment was constructed with PROMALS3D (http://prodata. swmed.edu/promals3d).

\section{Abbreviations}

AGUV: Aguacate virus; Be An 578142: Phlebovirus sp. Be An 578142; CP: Capsid protein; CFUV: Corfou virus; Co Ar 171616: Phlebovirus sp. Co Ar 171616; dsRNA: Double-stranded RNA; GOUV: Gouleako virus (unclassified bunyavirus); IWSV: Iranian wheat stripe virus; MSTV: Maize stripe virus; NC: Nucleocapsid; PTPV: Punta toro phlebovirus; RGST: Rice grassy stunt virus; RSV: Rice stripe virus; RdRp: RNA-dependent RNA polymerase; SFNV: Sandfly fever Naples virus; TUAV: Turuna virus; UHBV: Urochloa hoja blanca virus; UUKS: Uukuniemi virus; VP-161A: Phlebovirus sp. VP-161A; WYHV: Wheat yellow head virus.

\section{Competing interests}

The authors declare that they have no competing interests.

\section{Authors' contributions}

MK collected and analyzed the data; MK, WD and EVK interpreted the results and wrote the manuscript. All authors read and approved the final version.

\section{Acknowledgements}

EVK is supported by intramural funds of the US Department of Health and Human Services (to the National Library of Medicine).

\section{Author details}

'Department of Microbiology, Unité Biologie Moléculaire du Gène chez les Extrêmophiles, Institut Pasteur, Paris 75015, France. ${ }^{2}$ Department of Botany and Plant Pathology, Oregon State University, Corvallis, OR 97331, USA. ${ }^{3}$ National Center for Biotechnology Information, National Library of Medicine, National Institutes of Health, Bethesda, MD 20894, USA.

\section{Received: 9 February 2015 Accepted: 10 March 2015}

\section{Published online: 29 March 2015}

\section{References}

1. Dolja W, Koonin EV. Common origins and host-dependent diversity of plant and animal viromes. Curr Opin Virol. 2011;1(5):322-31.

2. Koonin EV, Dolja W. Virus world as an evolutionary network of viruses and capsidless selfish elements. Microbiol Mol Biol Rev. 2014;78(2):278-303.

3. Simmonds P. Methods for virus classification and the challenge of incorporating metagenomic sequence data. J Gen Virol. 2014, in press.

4. Sztuba-Solinska J, Urbanowicz A, Figlerowicz M, Bujarski JJ. RNA-RNA recombination in plant virus replication and evolution. Annu Rev Phytopathol. 2011:49:415-43.

5. Koonin EV, Wolf Yl, Nagasaki K, Dolja W. The Big Bang of picorna-like virus evolution antedates the radiation of eukaryotic supergroups. Nat Rev Microbiol. 2008;6(12):925-39.

6. Abrescia NG, Bamford DH, Grimes JM, Stuart DI. Structure unifies the viral universe. Annu Rev Biochem. 2012;81:795-822.
7. Bamford DH, Grimes JM, Stuart DI. What does structure tell us about virus evolution? Curr Opin Struct Biol. 2005;15(6):655-63.

8. Forterre P, Krupovic M, Prangishvili D. Cellular domains and viral lineages. Trends Microbiol. 2014;22(10):554-8.

9. Krupovic M, Bamford DH. Does the evolution of viral polymerases reflect the origin and evolution of viruses? Nat Rev Microbiol. 2009;7(3):250.

10. Raoult D, Forterre P. Redefining viruses: lessons from Mimivirus. Nat Rev Microbiol. 2008:6(4):315-9.

11. Krupovic M, Bamford DH. Order to the viral universe. J Virol. 2010;84(24):12476-9.

12. Koonin EV, Wolf YI, Nagasaki K, Dolja W. The complexity of the virus world. Nat Rev Microbiol. 2009;7:250.

13. Krupovic M, Bamford DH. Double-stranded DNA viruses: 20 families and only five different architectural principles for virion assembly. Curr Opin Virol. 2011;1(2):118-24.

14. Koonin EV. Evolution of double-stranded RNA viruses: a case for polyphyletic origin from different groups of positive-stranded RNA viruses. Semin Virol. 1992;3:327-39.

15. Koonin EV, Dolja W. Evolution and taxonomy of positive-strand RNA viruses: implications of comparative analysis of amino acid sequences. Crit Rev Biochem Mol Biol. 1993;28(5):375-430

16. Liu H, Fu Y, Xie J, Cheng J, Ghabrial SA, Li G, et al. Evolutionary genomics of mycovirus-related dsRNA viruses reveals cross-family horizontal gene transfer and evolution of diverse viral lineages. BMC Evol Biol. 2012;12:91.

17. Roossinck MJ, Sabanadzovic S, Okada R, Valverde RA. The remarkable evolutionary history of endornaviruses. J Gen Virol. 2011;92(Pt 11):2674-8.

18. Ghabrial SA. Origin, adaptation and evolutionary pathways of fungal viruses. Virus Genes. 1998;16(1):119-31.

19. Liu W, Chen J. A double-stranded RNA as the genome of a potential virus infecting Vicia faba. Virus Genes. 2009;39(1):126-31.

20. Martin RR, Zhou J, Tzanetakis IE. Blueberry latent virus: an amalgam of the Partitiviridae and Totiviridae. Virus Res. 2011;155(1):175-80.

21. Sabanadzovic S, Abou Ghanem-Sabanadzovic N, Valverde RA. A novel monopartite dsRNA virus from rhododendron. Arch Virol. 2010;155(11):1859-63.

22. Sabanadzovic S, Valverde RA, Brown JK, Martin RR, Tzanetakis IE. Southern tomato virus: the link between the families Totiviridae and Partitiviridae. Virus Res. 2009;140(1-2):130-7.

23. Wickner RB, Ghabrial SA, Nibert ML, Patterson JL, Wang CC. Family Totiviridae. In: King AMQ, Adams MJ, Carstens EB, Lefkowitz EJ, editors. Virus taxonomy: classification and nomenclature of viruses: Ninth Report of the International Committee on Taxonomy of Viruses. San Diego: Elsevier Academic Press; 2012. p. 639-50.

24. Ghabrial SA, Nibert ML, Maiss E, Lesker T, Baker TS, Tao YJ. Family Partitiviridae. In: King AMQ, Adams MJ, Carstens EB, Lefkowitz EJ, editors. Virus taxonomy: classification and nomenclature of viruses: Ninth Report of the International Committee on Taxonomy of Viruses. San Diego: Elsevier Academic Press; 2012. p. 522-34.

25. Isogai M, Nakamura T, Ishii K, Watanabe M, Yamagishi N, Yoshikawa N. Histochemical detection of Blueberry latent virus in highbush blueberry plant. J Gen Plant Pathol. 2011;77:304-6.

26. Söding J. Protein homology detection by HMM-HMM comparison. Bioinformatics. 2005;21(7):951-60.

27. Falk BW, Tsai JH. Biology and molecular biology of viruses in the genus Tenuivirus. Annu Rev Phytopathol. 1998;36:139-63.

28. Kormelink R, Garcia ML, Goodin M, Sasaya T, Haenni AL. Negative-strand RNA viruses: the plant-infecting counterparts. Virus Res. 2011;162(1-2):184-202.

29. Ferron F, Li Z, Danek El, Luo D, Wong Y, Coutard B, et al. The hexamer structure of Rift Valley fever virus nucleoprotein suggests a mechanism for its assembly into ribonucleoprotein complexes. PLoS Pathog. 2011;7(5):e1002030

30. Jiao L, Ouyang S, Liang M, Niu F, Shaw N, Wu W, et al. Structure of severe fever with thrombocytopenia syndrome virus nucleocapsid protein in complex with suramin reveals therapeutic potential. J Virol. 2013;87 (12):6829-39.

31. Raymond DD, Piper ME, Gerrard SR, Skiniotis G, Smith JL. Phleboviruses encapsidate their genomes by sequestering RNA bases. Proc Natl Acad Sci U S A. 2012;109(47):19208-13.

32. Liang D, Ma X, Qu Z, Hull R. Nucleic acid binding property of the gene products of rice stripe virus. Virus Genes. 2005;31(2):203-9.

33. Martins CR, Johnson JA, Lawrence DM, Choi TJ, Pisi AM, Tobin SL, et al. Sonchus yellow net rhabdovirus nuclear viroplasms contain polymeraseassociated proteins. J Virol. 1998;72(7):5669-79. 
34. Diemer GS, Stedman KM. A novel virus genome discovered in an extreme environment suggests recombination between unrelated groups of RNA and DNA viruses. Biol Direct. 2012;7:13.

35. Krupovic M, Koonin EV. Evolution of eukaryotic single-stranded DNA viruses of the Bidnaviridae family from genes of four other groups of widely different viruses. Sci Rep. 2014;4:5347.

36. Roux S, Enault F, Bronner G, Vaulot D, Forterre P, Krupovic M. Chimeric viruses blur the borders between the major groups of eukaryotic singlestranded DNA viruses. Nat Commun. 2013;4:2700.

37. Altschul SF, Madden TL, Schaffer AA, Zhang J, Zhang Z, Miller W, et al. Gapped BLAST and PSI-BLAST: a new generation of protein database search programs. Nucleic Acids Res. 1997;25(17):3389-402.

38. Pei J, Grishin NV. PROMALS3D: multiple protein sequence alignment enhanced with evolutionary and three-dimensional structural information. Methods Mol Biol. 2014;1079:263-71.

39. Waterhouse AM, Procter JB, Martin DM, Clamp M, Barton GJ. Jalview Version 2-a multiple sequence alignment editor and analysis workbench. Bioinformatics. 2009;25(9):1189-91.

40. Cole C, Barber JD, Barton GJ. The Jpred 3 secondary structure prediction server. Nucleic Acids Res. 2008;36(Web Server issue):W197-201.

41. Muhire BM, Varsani A, Martin DP. SDT: a virus classification tool based on pairwise sequence alignment and identity calculation. PLoS One. 2014;9(9):e108277.

42. Pettersen EF, Goddard TD, Huang CC, Couch GS, Greenblatt DM, Meng EC, et al. UCSF Chimera-a visualization system for exploratory research and analysis. J Comput Chem. 2004;25(13):1605-12.

43. Guindon S, Dufayard JF, Lefort V, Anisimova M, Hordijk W, Gascuel O. New algorithms and methods to estimate maximum-likelihood phylogenies: assessing the performance of PhyML 3.0. Syst Biol. 2010;59(3):307-21.

\section{Submit your next manuscript to BioMed Central and take full advantage of:}

- Convenient online submission

- Thorough peer review

- No space constraints or color figure charges

- Immediate publication on acceptance

- Inclusion in PubMed, CAS, Scopus and Google Scholar

- Research which is freely available for redistribution 